\title{
Los usos de las escalas espaciales y las prácticas historiográficas en el interior de la Argentina: un ejercicio de aproximación a partir del estudio de las políticas sociales
}

\author{
The uses of spatial scales and the historiographical practices within \\ Argentina: an approach exercise based on the study of social policies
}

\author{
María José Ortiz Bergia \\ ortizbergia.mj@gmail.com \\ Profesora asistente \\ Universidad Nacional de Córdoba \\ Av. Valparaíso, s/n - Ciudad Universitaria \\ 5000 - Córdoba Capital \\ Argentina
}

\section{Resumen}

En las últimas décadas, en la historiografía argentina han surgido polos de conocimiento desde el interior nacional que buscan articular alternativas a una producción académica generada en Buenos Aires. El propósito de este artículo es revisar las fortalezas y las debilidades de esas investigaciones analizando cómo construyen sus escalas espaciales de estudio. Este trabajo provee algunas respuestas preliminares a esta cuestión examinando la producción reciente dentro del campo de la historia social dedicada al estudio de las políticas sociales. El supuesto principal del trabajo es que muchos de los trabajos de este tipo suelen prestar escasa atención a la reflexión metodológica sobre sus marcos espaciales de investigación, limitando el alcance explicativo de sus trabajos y sus posibilidades de repensar el conocimiento historiográfico previo.

\section{Palabras claves}

Argentina; Fragmentación; Historia regional y local.

\begin{abstract}
In the past decades, new sources of knowledge from the interior of the country have emerged in the Argentine historiography, seeking to articulate alternatives to the academic production generated in Buenos Aires. The purpose of this article is to assess the strengths and weaknesses of these investigations, by analyzing how they construct their spatial scales of research. This paper provides some preliminary answers to this question, examining the recent production in the field of social history dedicated to social policies. The main assumption of this article is that these kinds of studies usually do not pay the due attention to the methodological reflection about their spatial scales of research. In that way, they reduce their explanatory scope and the possibilities of rethinking the previous historiographical knowledge.
\end{abstract}

Keywords

Argentina; Fragmentation; Regional and local history.

Recibido el: $22 / 6 / 2013$

Aprobado el: 16/9/2013 
A mediados de los años noventa, Luis Alberto Romero expresaba que "La historiografía argentina experimenta[ba] el mismo estallido de temas, perspectivas y paradigmas que caracteriza[ba] al resto de la comunidad académica occidental" (ROMERO 1996, p. 101). Quince años después los términos usados para describir el estado actual de la disciplina no difieren demasiado y la multiplicación constante de temáticas, perspectivas y problemas parece constituir un rasgo duradero del panorama académico argentino. ${ }^{1}$ Un correlato de ese contexto disciplinar es la proliferación de trabajos que adoptan delimitaciones espaciales diferentes a las nacionales, diversificando las aproximaciones a través de escalas locales, provinciales y regionales de investigación. Este último fenómeno está fuertemente vinculado con la multiplicación de los estudios descentrados geográficamente de los tradicionales polos historiográficos, con la emergencia de nuevos centros académicos en el interior del país, el crecimiento de profesionales $y$, como consecuencia, la elaboración paulatina de enriquecedoras miradas sobre el pasado nacional construidas desde y sobre las provincias. De tal modo, desde las últimas décadas, existe una importante proliferación de nuevos estudios desde ámbitos subnacionales que multiplican los relatos históricos sobre el pasado nacional, cuestionando y matizando los conocimientos historiográficos previos construidos desde ámbitos nacionales. En ese contexto resulta interesante reflexionar críticamente sobre los resultados alcanzados por esa producción, sus principales características, debilidades y fortalezas y su impacto en la reelaboración de los relatos históricos canonizados y la construcción de síntesis integradoras.

Desde esa perspectiva, en este trabajo nos hemos propuesto interrogarnos sobre cómo los historiadores dedicados a la producción histórica desde y sobre el interior del país delimitan espacialmente sus aproximaciones y qué consecuencias cognoscitivas derivan de esas decisiones en la comprensión histórica. Para volver viable este ejercicio hemos optado por abordar un eje de análisis centrado en un campo temático particular dentro de la historia social, el estudio de las políticas sociales, analizando los modos en que esas investigaciones operan con las escalas espaciales y cómo a partir de ellas se construyen los problemas de indagación. El trabajo, entonces, tiene como intención realizar una reflexión metodológica sobre una serie de rasgos y tendencias presentes en las prácticas disciplinares en el país, sin ambicionar generalizaciones, sino tan sólo incentivar discusiones sobre los modos de escritura de la historia.

Para realizar esta exploración hemos realizado una selección de trabajos presentados en eventos académicos que tradicionalmente agrupan a historiadores residentes en ámbitos provinciales, identificando aquellas ponencias relacionadas al estudio de las políticas sociales en la primera mitad del siglo XX en el interior del país. ${ }^{2}$ Cada una de esas instancias son eventos

\footnotetext{
${ }_{1}^{1}$ Para un diagnóstico reciente de este tipo, ver: (PAGANO 2010). Asimismo, como expresión de esta situación, se ha generado una interesante discusión a propósito de la magnitud que han adquirido las Jornadas Interescuelas/ Departamentos de Historia el evento de la disciplina más importante del país. (HISTORIAPOLITICA.COM, Dossier Interescuelas).

2 Jornadas Interescuelas/Departamentos de Historia: Tucumán, 2007 e Bariloche, 2009. Las Jornadas Nacionales de Historia Social: La Falda en 2007 e 2009. Las Jornadas de Historia de la Patagonia: Roca en
} 
que aúnan a investigadores de todo el país por lo que consideramos que son suficientemente representativos de lo que se está produciendo en el campo de las políticas sociales en la Argentina en la última década. Es necesario mencionar que este artículo no tuvo pretensiones de exhaustividad y, como consecuencia, algunas provincias han quedado fuera del análisis al no estar incluidas en los materiales que examinamos. Esto sucedió con las jurisdicciones de Catamarca, Formosa, Corrientes, Entre Ríos, San Luis, Tierra del Fuego y Misiones. La revisión de estos trabajos fue complementada con dos revistas periódicas que nos permitieron examinar trabajos publicados $y$, en consecuencia, de un mayor nivel de maduración. Para ello relevamos una revista argentina caracterizada por incorporar en sus páginas numerosas contribuciones provenientes del interior del país, el Anuario del Centro de Estudios Históricos (2001-2009). También hemos consultado la revista Entrepasados (1991-2009), publicación periódica con una notable gravitación en el campo historiográfico argentino y un referente en el tratamiento de los problemas relativos a la historia social.

\section{La historiografía argentina desde los años ochenta: profesionalización y crecimiento}

La multiplicación de las investigaciones provenientes del interior del país dentro de la producción historiográfica argentina debe comprenderse en el marco de las transformaciones más amplias sufridas en el campo desde el retorno a la democracia en los años ochenta. Así pues, luego de las consecuencias más adversas del quiebre académico de 1976 en el país se impuso una agenda de trabajo posdictadura consistente en actualizar la educación en el ámbito universitario y las investigaciones que se venían realizando, tendiendo a adoptar temáticas, interrogantes y propuestas metodológicas discutidas a nivel mundial. En ese contexto se generaron importantes avances en la renovación de los planes de estudio, con la (re)incorporación de profesores, muchos de los cuales se habían formado en el exterior, la realización de nuevas propuestas editoriales y la construcción de centros académicos en distintos puntos del país como resultado principalmente de la expansión del sistema universitario nacional (PAGANO 2010, p. 43). ${ }^{3}$

A grandes rasgos fue evidente el esfuerzo de los historiadores por adecuar sus prácticas a los parámetros disciplinares de sus pares internacionales (RODRIGUEZ 2002-2003; AGUILA 2012) y con ello la historiografía argentina avanzó significativamente en su profesionalización. A tres décadas del retorno a la democracia son evidentes los indicadores de la importante vitalidad de esta disciplina, con la multiplicación incesante de sus practicantes y la diversificación de sus propuestas temáticas, espaciales y temporales. Una expansión que no responde necesariamente a la existencia simultánea de varias "concepciones

2006, Santa Rosa en 2010. Las Primeras Jornadas Interdisciplinarias de Historia Regional: Mendoza en 2009. 3 Tres décadas después del inicio de esos procesos los resultados alcanzados han sido valorados de formas bastantes dispares. Principalmente es interesante la discusión entre quienes ponderan la profesionalización y consolidación disciplinar y quienes critican el profundo escepticismo existente sobre los usos públicos de la historia académica y lo que parece ser una evidente desconexión entre historia y política (CERNADAS; LVOVICH 2010, p. 19). 
historiográficas fuertes, sino de cierta heterodoxia teórica y metodológica" (SABATO 2001, p. 42).

Pero la proliferación de trabajos construidos desde escalas diferentes a la nacional, más allá de un resultado de los cambios disciplinares de las últimas décadas, también es una reacción contra ciertos rasgos de la historiografía argentina desde finales del siglo XIX. Al respecto, como explica Sandra Fernández, uno de los rasgos dominantes del campo histórico argentino a lo largo de décadas fue su propensión a generalizar conclusiones desde, en y sobre Buenos Aires, capital del país, al resto del territorio (FERNÁNDEZ 2007). Ese modo de operar de los historiadores tendió a generar una mirada empobrecedora del pasado nacional, al solapar o simplemente desconocer las experiencias pretéritas de buena parte de los argentinos, elevando a relato general los conocimientos alcanzados dentro de un recorte espacial limitado. La historia latinoamericana en general parece demostrar cierta propensión a escribirse desde estas perspectivas "nacionales". Según algunos autores, esto habría sido el resultado de un centralismo historiográfico "que negó lo heterogéneo y diverso, lo realmente existente en el nivel local y regional, para crear y justificar la creación de las historias nacionales, como síntesis de las nacientes identidades nacionales" (VIALES HURTADO 2010, p. 158; BERTRAND 2001; SERRANO ÁLVAREZ 2011).

En ese contexto disciplinar, el despliegue paulatino de nuevos polos de construcción del conocimiento histórico busca descentrar esa mirada sobre la capital del país y visibilizar trayectorias históricas divergentes, con interpretaciones y periodizaciones discordantes de los procesos históricos nacionales. Probablemente, en las últimas dos décadas estas constituyen algunas de las "notas más salientes de la historiografía reciente" al permitir identificar discontinuidades en los procesos sociales a lo largo del país y resignificar la producción historiográfica previa (PAGANO 2010, p. 61).

Un relevamiento de los centros de producción dedicados a la historia social y, en especial, a las políticas sociales en el interior del país puede resultar instructivo sobre esa dispersión introducida en el campo histórico en las últimas décadas en la Argentina. Por cuestiones de espacio aquí solamente pasamos revista a los referentes académicos más relevantes dentro de la historia social argentina contemporánea en el interior del país. ${ }^{4}$

Inicialmente, puede resultar analíticamente útil distinguir dos ejes alrededor de los cuales se han desarrollado grupos de historiadores interesados por la historia social entre los años ochenta y los noventa del siglo pasado en la Argentina. El primero se vincula estrechamente con el desarrollo de la historia urbana en el período de entre siglos (XIX-XX) y ha estado centrado en los fenómenos englobados bajo el término "cuestión social", la inmigración y el movimiento obrero en ciudades como Buenos Aires, Rosario y Córdoba, espacios urbanos insertos en el desarrollo agro exportador del país. Es posible 
identificar otra línea, en cambio, más relacionada con las derivas historiográficas generadas dentro de la historia económica y los estudios regionales. Esta última se ha dedicado a analizar a los trabajadores y las condiciones de vida alrededor de economías regionales como la azucarera y la vitivinícola.

Desde los años ochenta un espacio de notable renovación historiográfica en el interior del país se desarrolló alrededor de la Universidad Nacional de Rosario. En esa institución los estudios sobre estas temáticas se han centrado en los problemas relativos al mundo del trabajo para luego incluir otros intereses como el asociacionismo y las prácticas sociales y políticas de la población en general. Los nombres de Ricardo Falcón, Marta Bonaudo y Sandra Fernández remiten a diferentes generaciones de historiadores que propiciaron revisiones en la historiografía social a nivel nacional y provincial, especialmente, incorporando discusiones sobre lo regional en la comprensión de la espacialización de las prácticas sociales. Con una trayectoria similar se puede reconocer la producción en historia social desarrollada en Córdoba. Inicialmente centrada en el mundo del trabajo y representada por la producción de Ofelia Pianetto, este campo de estudios se ha extendido a las prácticas asociativas, de consumo y a los procesos de salud y enfermedad a través de investigadores como Beatriz Moreyra, Fernando Remedi y Adrián Carbonetti. Desde un contexto similar, otro polo de construcción de estudios sobre historia social se ha desarrollado sobre la costa argentina, en la Universidad Nacional de Mar del Plata. En esa institución se ha destacado la historiadora Elisa Pastoriza quien ha realizado sustanciales aportes al conocimiento de las políticas sociales durante el peronismo, los trabajadores, el ocio y el turismo. Las indagaciones sobre la historia de la inmigración, el asociacionismo, el trabajo marítimo y los procesos de salud-enfermedad constituyen también allí tópicos destacados entre los historiadores agrupados en esa casa de estudios.

En el norte del país los espacios de producción en torno a la historia social más importantes se pueden identificar alrededor de la Universidad Nacional de Tucumán. Estos se han desarrollado a partir de una fuerte preocupación por los problemas relativos a los procesos de producción azucareros y a las economías regionales. Las investigaciones de Daniel Campi y Celia Bravo inauguraron una amplia cantera de indagaciones en torno al desarrollo socioeconómico del Noroeste argentino y a las particularidades del mundo del trabajo azucarero, el movimiento obrero y sus condiciones de vida. Desde una lógica similar a esta, otro polo de interés es la producción generada en conexión con los estudios regionales en la Universidad Nacional del Comahue. En ese marco institucional es necesario destacar la figura de Enrique Masés quien promovió la comprensión del mundo del trabajo en Neuquén y en la región norpatagónica.

También en el contexto particular que proporcionan los Territorios Nacionales, ${ }^{5}$ en la provincia de La Pampa, desde comienzo de los años noventa,

\footnotetext{
${ }^{5}$ Los Territorios Nacionales fueron creaciones político-administrativas a través de los cuales se incorporaron los espacios ocupados por el Estado argentino luego de las campañas militares realizadas sobre las poblaciones indígenas del sur y del noreste en el último cuarto del siglo XIX. Estos espacios recién comenzaron a convertirse en provincias a mediados del siglo XX.
} 
se han desplegado investigaciones pioneras relativas a la historia de las mujeres y el género, proporcionando una enorme vitalidad a los estudios históricos sobre las políticas sociales a través de indagaciones como las de María Herminia Di Liscia y María Silvia Di Liscia. Varias líneas de trabajo se abordaron desde entonces en la Universidad Nacional de La Pampa orientadas a los sistemas de educación y a las intervenciones sanitarias en los territorios nacionales.

La revisión anterior no ha tenido pretensiones de exhaustividad, sino el propósito de identificar algunos de los núcleos de producción con mayor gravitación desde los años noventa en el interior del país. Consideramos que esa multiplicación de los centros universitarios dedicados a la historia social alienta una reflexión sobre los modos en que se construyen ese tipo de investigaciones y cuáles son sus posibilidades de proporcionar revisiones a los conocimientos historiográficos previos.

\section{¿Cómo se escribe la historia en el interior país?}

La importancia de los cambios relevados en las páginas previas nos impulsó a intentar una reflexión respecto a cómo se elaboran las investigaciones en el campo de la historia de las políticas sociales desde y sobre el interior del país y cómo las mismas se vinculan con aquellas que adoptan escalas de dimensión nacional. En otras palabras, nos interesó conocer cómo los historiadores delimitan y construyen sus objetos de estudio y qué consecuencias se extraen de esas opciones. Con esa finalidad en el trabajo intentamos identificar cuáles son las escalas espaciales más frecuentes entre los historiadores del interior del país a la hora de definir sus problemas de investigación, cuáles son las fundamentaciones metodológicas que sustentan esas elecciones y de qué modo se vincula su producción con aquella realizada a partir de otras delimitaciones espaciales como las nacionales.

Inicialmente podemos afirmar que entre los trabajos analizados existe una tendencia persistente caracterizada por construir investigaciones a partir de ámbitos provinciales, urbanos y territorios nacionales, en otras palabras, delimitaciones recortadas en forma generalizada en base a jurisdicciones político-administrativas. Más recurrentemente, entonces, municipios, departamentos, partidos, provincias y territorios nacionales, son los recortes elegidos en contraposición a lo nacional. Pero, más allá del descredito al que ese tipo de delimitaciones ha dado lugar, al imponer supuestamente demarcaciones artificiales a los procesos históricos, ${ }^{6}$ lo que llama nuestra atención es que en forma frecuente las mismas no suelen acompañarse por una reflexión sistemática sobre las consecuencias metodológicas del problema así construido.

\footnotetext{
${ }^{6}$ Frecuentemente los profesionales identificados con la "historia regional" suelen desdeñar estas formas de delimitar los objetos de estudio dado que resultan poco apropiados para aprehender determinados procesos históricos sociales y económicos. Sin embargo, resulta necesario reflexionar que la validez del recorte político-administrativo depende de los problemas de investigación planteados por los historiadores y no puede ser desplazado porque sí. Como expresión de ello, Favaro y Scutti realizan una aproximación a la historia política de la norpatagonia desde un abordaje de tipo regional que recupera la escala provincial porque como las autoras afirman "desde la región norpatagónica, es posible escribir historia política, con una dimensión, la provincial, que contribuya a la historiografía nacional, sin que ello sea, factual, sumatoria de hechos o historia institucional" (FAVARO; SCURI 2003, p. 12).
} 
Por el contrario, los autores suelen limitarse a indicar el lugar geográfico en el que se proponen ubicar sus estudios, rehuyendo explicitar los fundamentos de tal elección y sus posibles efectos sobre el conocimiento así elaborado. De tal modo, son muy exiguos los trabajos en los que el investigador se reconozca explícitamente construyendo sus escalas de estudio (FERNÁNDEZ; DALLA CORTE 2001; FERNÁNDEZ 2006; 2007) y clarifique los criterios de su utilización, el por qué para determinados problemas adoptó un recorte y por qué el mismo se considera metodológicamente más conveniente que otros.

Esto último merece destacarse porque parece existir una cierta desaprensión frente a las consecuencias cognoscitivas de las delimitaciones espaciales, eludiendo la constatación de que los recortes espaciales, sin más fundamentos que el capricho del historiador, pueden tener consecuencias importantes en el valor interpretativo de las conclusiones construidas. El despliegue territorial de determinadas prácticas puede pasarse por alto, generando comprensiones sesgadas sobre los problemas de investigación e invisibilizando posibles rupturas y disrupciones, evidentes desde otras escalas de estudio. ${ }^{7}$ En otras palabras, no existe una consideración especial sobre cómo la delimitación espacial de un problema y la variación de la escala de observación es una decisión metodológica antes que temática.

Esto último se comprende mejor si tenemos en cuenta que una escala es una categoría que se adopta con la finalidad de aprehender una realidad y lograr representarla. En otras palabras, el historiador al optar por una escala está tomando una decisión explícita respecto a la cantidad y al tipo de información que considera adecuada para alcanzar su propósito. En consecuencia no existe una relación lineal entre lo que el historiador se propone estudiar y las modalidades más apropiadas que tiene para representarlo (PONS; SERNA 2007 , p. 25). Por el contrario, la elección de una escala es una estrategia de indagación derivada de los objetivos de los profesionales, "la relación entre los puntos que plantea discutir y la pertinencia de las elecciones metodológicas para estudiar ese tema" (BARRIERA 2006, p. 20). De tal modo, la sola delimitación de un ámbito subnacional sin una adecuada reflexión sobre sus efectos en la operación historiográfica reduce significativamente el potencial analítico de las investigaciones realizadas.

Resulta interesante constatar que esa escasa reflexión sobre las delimitaciones espaciales no es necesariamente una particularidad de la historiografía argentina. Peter Stearns ha destacado como un rasgo generalizado la tendencia de los historiadores sociales a ser poco explícitos sobre los recortes espaciales de sus estudios, prestando escasa atención a sus coordenadas geográficas en comparación a su tradicional apego a las temporales (STEARNS 2006). De tal modo, los historiadores parecen encontrarse muy poco adaptados, por rutina, comodidad o formación, a adoptar nuevas herramientas espaciales y a modificar sus prácticas al delimitar sus trabajos. El impacto de estas

\footnotetext{
7 Específicamente, en un trabajo reciente Juan Cerdá realiza un experimento empírico destinado a mostrar cómo las escalas espaciales de estudio permiten aprehender o no ciertos procesos históricos a partir de recortes regionales y provinciales (CERDÁ 2009).
} 
discusiones y su relevancia actual se comprende mejor dentro de los debates contemporáneos relativos al valor cognoscitivo de las perspectivas microanalíticas y a las novedades introducidas por la historia trasnacional. ${ }^{8}$ Ambas propuestas coinciden en que la delimitación espacial de los problemas abordados constituye una decisión central de la operación historiográfica, porque al permitir superar los marcos nacionales visibiliza singularidades y conexiones poco aprehendidas previamente.

Una segunda cuestión que atrajo nuestra atención al analizar la producción en historia social desde y sobre el interior del país fueron las relaciones que suelen establecerse entre esas historias elaboradas desde el interior nacional y aquellas construidas en la Capital Federal. Al respecto, un rasgo recurrente que hemos podido identificar en las investigaciones revisadas es la tendencia a adoptar temáticas, problemas y categorías en forma acrítica de la agenda historiográfica porteña para incorporarlas a los estudios centrados en otros ámbitos geográficos. Ya en los años noventa Sandra Fernández y Gabriela Dalla Corte mencionaban que uno de los rasgos que caracterizaban a esa producción histórica era esta propensión a recuperar los problemas abordados por la historiografía centrada en Buenos Aires, trasladándolos a otros marcos espaciales con la finalidad de comprobar su pertinencia "nacional" (DALLA CORTE; FERNADEZ 1997-1998). Un diagnóstico similar menciona Carlos Forcadell Álvarez para la historiografía española, en donde ciertos trabajos de historia local o regional parecen limitarse al rol de monografías históricas que intentan captar cómo los procesos generales se proyectan en espacios acotados, práctica que corre el riesgo de convertirse en un simple ejercicio de erudición (FORCADELL ÁLVAREZ 1995-1996, p. 21). Muchos de los trabajos relevados en este artículo despliegan también esa práctica que supone una actitud poco crítica frente al registro histórico. Algunos estudios parecen limitar sus ambiciones a verificar el alcance de los conocimientos realizados en indagaciones centradas en Buenos Aires y a comprobar su validez a través de investigaciones empíricas exhaustivas de contextos distintos para los que fueron pensados.

Esta descontextualización de los problemas bajo estudio genera una comprensión limitada de los procesos históricos bajo la lente. Para ser más gráficos, en las investigaciones relevadas es recurrente la utilización de términos como "cuestión social", "cuestión obrera", "crisis hospitalaria" o "reformismo liberal" para hacer referencia a procesos históricos indeterminados, pasando por alto las realidades empíricas concretas a las que esos términos hacen referencia. Resulta innegable, por el contrario, que los procesos sociales que durante el período de entreguerras atravesaron a algunas ciudades del área litoral, mejor insertas en el proceso de desarrollo económico de finales del siglo XIX y comienzos del XX, difícilmente puedan asimilarse acríticamente con los que afectaron a espacios como los Territorios Nacionales integrados muy tardíamente al esquema político-institucional y económico nacional.

\footnotetext{
8 En esos términos, son continuas las críticas por lo mal adaptados que se encuentran actualmente los historiadores para adoptar problemas de investigaciones en escalas trasnacionales dado que por tradición escolar sus parámetros geográficos siguen siendo los nacionales (CROSS 2006, p. 631).
} 
En consecuencia, términos como "cuestión social" no remiten a realidades evidentes y se torna necesario identificar claramente los referentes empíricos con los que se trabaja. Una expresión evidente también de esta práctica es la de privilegiar los problemas construidos desde espacios urbanos, cuando una de las particularidades principales de los espacios del interior del país en la Argentina es la tensión permanente entre lo urbano y lo rural. En muchos de los estudios elaborados se tienden a pasar por alto las interrelaciones entre esos dos ámbitos, enfatizando las trayectorias y experiencias de los actores urbanos en espacios con contornos predominantemente rurales. Incluso, una práctica común de muchos de las investigaciones revisadas es la de iniciar los estudios con un sucinto esquema histórico en donde se destaca la particularidad del objeto analizado y sus diferencias respecto a la ciudad de Buenos Aires y al "litoral". No obstante, ese marco previo deviene solamente un mecanismo con el cual generar "efectos de realidad", antes que sustentar explicaciones o interpretaciones históricas, dado que en el cuerpo de la investigación es rápidamente marginado del análisis (REVEL 2005, p. 52). Lo que queremos subrayar con estos ejemplos es que esa propensión a apropiar conceptos e interpretaciones en forma poco reflexiva, buscando los elementos comunes con la historia porteña, puede generar la negación misma de las posibilidades de acceso al pasado. ${ }^{9}$ Es necesario hacer hincapié en que la investigación histórica no constituye una operación hipotético-deductiva, sino una actividad intelectual destinada a historizar las formas sociales en su especificidad. En consecuencia al utilizar una agenda previamente estructurada los historiadores tienden a identificar más frecuentemente los elementos comunes antes que a explicar lo singular y lo particular.

Finalmente, también existe otra consecuencia que es necesario destacar de estas formas de operar con el conocimiento histórico. Esta propensión, a enfatizar las semejanzas antes que a explotar significativamente las diferencias y las especificidades, reduce en buena parte el potencial explicativo de los abordajes que adoptan delimitaciones diferentes a la nacional. Sobre esta cuestión ya hemos mencionado la habitual falta de discusión sobre las consecuencias intelectuales de las delimitaciones espaciales con las que se construyen las investigaciones. Esto último nos alerta sobre la necesidad de ponderar más acabadamente los efectos cognoscitivos de las escalas espaciales y la necesidad de explotar más significativamente el potencial explicativo de los contextos históricos delimitados desde ámbitos subnacionales.

\section{Construyendo una nueva agenda, las escalas espaciales como rupturas}

Nos parece importante destacar la importancia de reflexionar sobre las escalas espaciales porque consideramos que son los rasgos distintivos de los contextos locales, lo específico y lo particular del interior en el marco global, lo que valida y activa los aspectos más enriquecedores de las aproximaciones 
desde y sobre los espacios provinciales, al permitir "detectar las peculiaridades de cada proceso, [...] observar las semejanzas y las diferencias regionales, las continuidades y las rupturas" (ARECES 2008, p. 27). En otras palabras, el abordaje de los problemas históricos a partir de escalas provinciales, regionales o locales, enriquece la historia nacional al mostrar los límites de sus proposiciones y al permitir eludir las miradas uniformes y lineales sobre los procesos históricos. Los estudios elaborados desde esas escalas tienen el potencial de mostrar cómo la espacialización de los fenómenos históricos adopta modalidades y periodizaciones específicas según los contextos.

El planteo anterior se vincula con uno de los fundamentos más importantes de las perspectivas microanalíticas que sostienen la necesidad de avanzar en investigaciones que no busquen la comprobación de hipótesis, sino lo irrepetible que hace única una experiencia pretérita. Esto implica una verdadera revisión de nuestra manera de trabajar con modelos. La finalidad del historiador no debe ser su comprobación ni su verificación, sino evidenciar sus límites y propiciar su problematización. Sobre este punto, Michel de Certeau diría que lo particular tiene como función introducir un interrogante, dando cuenta de todo aquello que queda fuera del modelo (DE CERTEAU 1993, p. 101). Del mismo modo, los diferentes recortes espaciales que elaboran los historiadores, sean estos nacionales, provinciales, locales o regionales, deberían ser vías con las que revisar, complejizar y cuestionar los conocimientos historiográficos previos propiciando otros nuevos.

Dentro de la producción historiográfica que hemos analizado es posible identificar varios estudios enriquecedores que adoptan estas modalidades de trabajo y que tenemos la inquietud de destacar. Los mismos se caracterizan por prestar una singular atención al peso de los contextos específicos con la finalidad de alcanzar una comprensión más amplia de las discontinuidades de los procesos históricos a lo largo del país.

Como expresiones de este fenómeno en la historia social merecen destacarse algunos abordajes recientes sobre la problemática de las políticas laborales en los años treinta que realizan interesantes revisiones a los desarrollos historiográficos previos. Desde una serie de investigaciones elaboradas en los años ochenta como las de Ricardo Gaudio y Jorge Pilone, en la historiografía argentina se asumió que la mayor intervención estatal en las relaciones laborales en los años treinta del siglo XX había constituido una reacción al incremento de la movilización obrera en un contexto de intensificación del desarrollo industrial por sustitución de importaciones (GAUDIO; PILONE 1983). Sin embargo, recientemente, investigaciones situadas en Santa Fe y Tucumán, han podido identificar para esas provincias procesos similares de creciente intervención estatal en lo laboral, pero en contextos diferentes a los que sustentaron las aproximaciones previas centradas en la ciudad de Buenos Aires y en el Conurbano Bonaerense (PIAZZESI 2009; ULLIVARRI 2009). Esos estudios han identificado nuevas variables explicativas para dar cuenta de las políticas laborales creadas en esa coyuntura, más precisamente, han subrayado la incidencia de nuevas formas de legitimación política-estatal para explicar esas innovaciones institucionales. 
Esto supone enfatizar variables que habían sido solapadas o minimizadas en los análisis previos, mostrando la necesidad de incorporar la variable regional en la comprensión del proceso de construcción del intervencionismo estatal. De ese modo, esos estudios, atentos a la importancia de revisar desde espacios provinciales los relatos canonizados del pasado nacional, incorporan nuevos abordajes, comentarios y discusiones a los conocimientos existentes.

Desde esa misma lógica una perspectiva que ha crecido enormemente en los últimos años y resulta verdaderamente estimulante, es la producción elaborada alrededor de los Territorios Nacionales, en especial de aquellos situados en la frontera sur.

Existe un buen número de ejemplos en las recientes dos décadas que vuelven evidente la potencialidad de las investigaciones sobre los Territorios Nacionales para revisar las prácticas disciplinares. Una referente indiscutida en esta tradición ha sido Susana Bandieri, quien desde los años ochenta ha impulsado una práctica importante de discusión sobre las formas de pensar los Territorios en el marco de una historiografía "nacional" calificada de uniformadora. ${ }^{10}$ Sería necesario agregar que estas reflexiones no sólo han incentivado revisiones en el ámbito de la historia económica sino también en otros campos como los de la historia política y la historia social. En particular en los últimos años, investigaciones como las de Martha Ruffini, Mario Arias Buccarelli, Orietta Favaro y Lisandro Gallucci, estimulan la reflexión creciente sobre cuestiones como las prácticas políticas, la estatidad y la ciudadanía en contextos históricos divergentes a los que tradicionalmente habían fundamentado las reflexiones argentinas, las catorce provincias históricas y la Capital Federal (RUFFINI 2000; FAVARO; ARIAS BUCCARELLI 1995; FAVARO; SCURI 2003; GALLUCCI 2005). Esos problemas son redimensionados y vueltos a discutir desde la perspectiva de los Territorios Nacionales, elaborando y proponiendo nuevas periodizaciones, explicaciones y descripciones de los procesos históricos previos. Bandieri es especialmente enfática sobre las posibilidades que tienen esas historias regionales de romper con los relatos nacionales generalizados al conjunto de la sociedad (BOHN MARTINS 2009).

Algo similar ha sucedido en lo que respecta a la historia social. Las pesquisas sobre las políticas sociales generadas sobre La Pampa a comienzos del siglo XX, desde el contexto específico de su condición de Territorio Nacional, han permitido a investigadoras como María Silvia Di Liscia discutir el alcance de determinados fenómenos, como los de la "medicalización" y la extensión de mecanismos de "control social", para entender los procesos históricos de construcción de las políticas sociales y del Estado social en la Argentina de la primera mitad del siglo XX. Esto implica destacar discontinuidades en el despliegue de las políticas sociales, limitaciones y cambios en las periodizaciones sobre su desarrollo. Asimismo, sus debates sobre la eugenesia han puesto en entredicho la influencia de estos saberes en las políticas públicas y la eficacia

\footnotetext{
10 Son numerosos los trabajos donde Susana Bandieri ha explicado su experiencia personal y colectiva de acercamiento a la historia regional y el valor explicativo que esta perspectiva le proporcionó para revisar la supuesta orientación atlántica del mercado nacional (BANDIERI 2007; 2008).
} 
de esas intervenciones para regular la vida de la población. Finalmente, desde las particularidades de los Territorios Nacionales, Di Liscia ha propuesto una periodización distinta sobre la expansión de los servicios de salud, diferenciando la experiencia de una ciudad como Buenos Aires, con una infraestructura de servicios médicos significativos para el período de entreguerras, de un espacio como el de La Pampa, donde la escasez de elementos para la lucha sanitaria fue la norma a lo largo de la primera mitad del siglo XX (BOHOSLAVSKY; DI LISCIA 2008; DI LISCIA 2008a; 2008b). Al respecto la autora ha destacado que

las políticas a nivel nacional repercuten [...] de manera desigual en las áreas del interior, sobre todo en los Territorios Nacionales. Estas regiones constituyen ámbitos políticos con un desarrollo paralelo y a la vez, diferente a las provincias históricas, con escasa población urbana, incipiente administración pública y sin participación electoral salvo en el ámbito municipal y nacional, entre otras características distintivas (DI LISCIA 2007, p. 95).

En pocas palabras, ha permitido revisar algunos supuestos construidos desde Buenos Aires que difícilmente eran generalizables al resto del país como la temprana instalación de un sistema de servicios públicos de salud y el éxito de los lenguajes poblacionales en el diseño e implementación de las políticas sanitarias. Esos trabajos muestran las limitaciones existentes en los supuestos historiográficos construidos previamente y propone problematizarlos a través de la singularidad que plantea la experiencia histórica de los Territorios Nacionales en donde la expansión de los aparatos estatales fue extremadamente dificultosa y lenta.

Estasaproximacionesmerecen destacarse porlas inquietudes metodológicas, temáticas y epistemológicas que proponen a los historiadores que escriben desde el interior del país y sobre el interior del país. Esta producción impulsada a partir del reconocimiento de la diferencia respecto a las proposiciones de la historia nacional, busca construir una historia más compleja antes que uniformar y homologar, de asimilar todas las historias a una. Muestra de tal modo que la reducción de la escala no se agota en la confirmación de lo general, como un "reflejo pasivo de algo más vasto" (PONS; SERNA 2004, p. 23). Por el contrario, la misma permite multiplicar la agenda de problemas de la historia social, obviando la comprobación de hipótesis y poniéndolas en discusión, evitando las simplificaciones y favoreciendo la diversidad de perspectivas (BONAUDO 2008).

Ahora bien, las reflexiones anteriores nos llevan a cuestionarnos sobre cómo en el largo plazo es necesario plantear la integración de esas miradas tan complejas sobre los procesos históricos nacionales. Esta problemática debe comprenderse en un contexto en el que, al igual que en el ámbito internacional, se reconoce que la fragmentación constituye uno de los rasgos permanentes del estado de los estudios históricos. Es posible afirmar que el campo historiográfico en su conjunto ha atravesado varias décadas de un continuo crecimiento "generoso pero descontrolado", en donde si bien la multiplicación de objetos, temáticas y metodologías innovadoras ha permitido iluminar algunas facetas olvidadas por la historia estructural, a su vez, la continua fragmentación de los saberes 
ha sido acompañada por la ausencia de síntesis explicativas y de consensos disciplinares que permitan aglutinar los diferentes proyectos historiográficos (REVEL 1996, p. 18; DA SILVA 2005, p. 160).

Más específicamente, en el caso argentino, muchos investigadores han afirmado que en las últimas décadas el enorme crecimiento de la producción historiográfica ha favorecido una historia "coralina" en la que resulta cada vez más arduo reconocer elementos comunes y núcleos articuladores. Roy Hora y Javier Trímboli en los noventa destacaban que la historiografía argentina crecía en parcelas en las que faltaba una trama que estructurara encadenamientos y conexiones entre trabajos discontinuos, práctica que generaba el peligro de "resignarse al fragmento, a la imagen desconectada, a la baja tensión con el pasado que evoca, puede condenarse a perder capacidad de interpretación y de significación" (HORA; TRIMBOLI 1994, p. 96). Comentando el estado de la historia social, también, Fernando Remedi señalaba que en la Argentina la especialización ha venido sufriendo un proceso de paulatina reducción de su posicionamiento dentro del campo historiográfico a lo que se sumaba una "gran heterogeneidad y, sobre todo, una notable dispersión, observándose la ausencia de núcleos aglutinantes, articuladores, y de cualquier intento de contribuir a la elaboración de alguna visión de conjunto, que ofrezca una síntesis siquiera de ciertos aspectos o sectores de la sociedad" (REMEDI 2011, p. 57). En términos generales estos balances generan un cuestionamiento a la multiplicación de perspectivas temáticas, temporales y espaciales producida en los últimos tiempos y evalúan negativamente sus consecuencias en la construcción de síntesis explicativas del cambio histórico.

La respuesta más adecuada a la tensión existente entre esa necesidad de síntesis y la actual fragmentación no puede ser absoluta, sino más bien el resultado de una mediación, en que la adopción de propuestas más integradoras no implique automáticamente una abdicación de las aproximaciones que buscan mostrar experiencias históricas diversas y heterogéneas a lo largo del tiempo y del espacio. ${ }^{11}$ Esto supone incentivar síntesis cautelosas en la construcción de sus generalizaciones, con un control permanente sobre si son efectivamente el resultado de una operación comparativa sistemática o sólo el producto de una operación uniformadora anuladora de las discontinuidades (BENDER 2002, p. 136). En otras palabras, esto implica aportar inteligibilidad al conjunto de las contribuciones realizadas por la historiografía argentina sin por ello adoptar el escasamente productivo camino de la historia agregativa o la subordinación a una tendencia homogeneizadora nacional (DALLA CORTE; FERNÁNDEZ 1997-1998; KINDGARD 2002, p. 68). En definitiva, es necesario proporcionar configuraciones interpretativas novedosas que adopten como dato permanente la heterogeneidad espacial de los procesos históricos.

\footnotetext{
${ }_{11}$ Dentro del debate estadounidense los reclamos por síntesis han sido ponderado no necesariamente en forma positiva, dada la posición de algunos historiadores que afirman que la fragmentación de perspectivas históricas es uno de los logros de la historiografía contemporánea (MONKKONEN 1986, p. 1154).
} 


\section{Recapitulando}

De la lectura de la producción de los historiadores sociales del interior del país nos surgen una serie de reflexiones que creemos enriquecedoras para pensar el estado de actual de la disciplina. Primero, resulta evidente que existe en muchos casos entre la producción histórica relevada cierta tendencia a rehuir la discusión y la reflexión sobre las dimensiones espaciales a partir de las cuales se elaboran los problemas de trabajo. Reiteradamente, lo nacional, lo provincial, lo local y lo regional parecen insertarse como simples "escenarios" sin derivaciones cognoscitivas importantes, operación que pasa por alto los cambios en la observación que los mismos suponen y la importancia de los contextos en la interpretación histórica. También como ya han marcado otros investigadores, existe cierta tendencia bastante extendida hacia la inclusión de las discusiones de la historia porteña buscando verificar su pertinencia para contextos espaciales diferentes, insistiendo en los elementos comunes y soslayando en gran parte los rasgos singulares de las trayectorias locales. Destacamos esta última característica porque la misma tiene consecuencias importantes en los conocimientos producidos, al reducir las posibilidades de visibilizar y explicar la complejidad histórica del territorio y rediscutir los conocimientos elaborados previamente. En otras palabras, estas operaciones diluyen el potencial cuestionador de las escalas espaciales para iluminar aspectos nuevos de los procesos históricos y revisar los supuestos canónicos.

Frente a estos rasgos, los debates sobre las escalas espaciales y el valor de algunos trabajos llevan a pensar que la opción debería ser distinta. Los estudios históricos en el interior nacional no se justifican por su valor agregativo y de verificación de la historia nacional producida desde Buenos Aires y generalizada acríticamente, sino por todo aquello que complejizan y ofrecen al conocimiento social. Para ello partimos de la constatación de que las delimitaciones espaciales constituyen opciones metodológicas que inciden en el conocimiento elaborado. De ese modo, el abordaje histórico a partir de lo regional, lo provincial o lo local no supone inscribir nuestras producciones como complementos, matizaciones o agregados de una historia mayor, sino como proyectos tendientes a multiplicar y expandir las preguntas válidas y las posibles respuestas. Esto se basa en el supuesto que asume que no existen vías privilegiadas en la exploración del registro histórico y, por el contrario, múltiples escalas de observación permiten producir nuevos conocimientos al cambiar la lente y la posibilidad de acceder a diferentes niveles de información sobre los procesos sociales (ROSENTAL 1996, p. 143).

\section{Referencias bibliográficas}

ARECES, Nidia R. La Etnohistoria y los estudios regionales. Andes, Salta: UNAS, n. 19, p. 15-28, 2008.

BANDIERI, Susana. La dimensión regional como alternativa analítica para pensar otros espacios y nuevas periodizaciones. In: BANDIERI, Susana; BLANCO, Graciela; BLANCO, Mónica (coord.). Las escalas de la historia comparada. Tomo 2: Empresas y empresarios. La cuestión regional. Buenos Aires: Miño y Dávila Editorial, 2008, p. 311-333. 
Nuevas investigaciones, otra historia la Patagonia en perspectiva regional. In: FERNÁNDEZ, Sandra (comp). Más allá del territorio: la historia regional y local como problema. Discusiones, balances y proyecciones. Rosario: Prohistoria, 2007. p. 47-71.

BARRIERA, Darío G. Escalas de observación y prácticas historiográficas. La construcción de horizontes alternativos de investigación. In: DALLA CORTE, Gabriela; GARCÍA JORDÁN Pilar; LUNA, Lola G. et al. (coord.). Homogeneidad, diferencia y exclusión en América: $X$ Encuentro-Debate América Latina ayer y hoy. Barcelona: Universitat de Barcelona, 2006, p. 15-38.

BENDER, Thomas. Strategies of Narrative Synthesis in American History. American Historical Review, Indiana: American Historical Association, v. 107, n. 1, p. 129-153, 2002.

BERTRAND, Michel. Histoire et régions dans l' historiographie américaniste: réflexions á propos $d^{\prime}$ une association féconde. Avances del Cesor, Rosario: ISHIR/CONICET, n. 3, p. 7-16, 2001.

BOHN MARTINS, Maria Cristina. A história regional e a historiografia Argentina: entrevista com Susana Bandieri. História Unisinos, São Leopoldo: Universidade do Vale do Rio dos Sinos, v. 13, n. 1, p. 96-102, 2009.

BOHOSLAVSKY, Ernesto; DI LISCIA, María Silvia. La profilaxis del viento. Instituciones represivas y sanitarias en la Patagonia argentina, 18801940. Asclepio: Revista de Historia de la Medicina y de la Ciencia, Madrid: CSIC, v. LX, n. 2, p. 187-206, 2008.

BONAUDO, Marta. Otra vez la "Fantásmatica" historia regional. In: BANDIERI, Susana; BLANCO, Graciela; BLANCO, Mónica (coord.). Las escalas de la historia comparada. Tomo 2: Empresas y empresarios. La cuestión regional. Buenos Aires: Miño y Dávila Editorial, 2008, p. 227-231.

CERDÁ, Juan Manuel. Sociedades dinámicas y territorios flexibles. Primeras Jornadas Interdisciplinarias de Investigaciones Regionales, Mendoza: UNCuyo, CD-Rom, 2009, s/p.

CERNADAS, Jorge; LVOVICH, Daniel. Historia, ¿para qué? Revisitas a una vieja pregunta. Buenos Aires: Prometeo, UNGS, 2010.

CROSS, Gary. Crowds and Leisure: Thinking Comparatively across the 20th Century. Journal of Social History, George Mason University, v. 39, n. 3, p. 631-650, 2006.

DA SILVA, Fernando Teixeira. História e Ciências Sociais: zonas de fronteira. História, São Paulo: Universidade Estadual Paulista Júlio de Mesquita Filho, v. 24, n. 1, p. 127-166, 2005.

DALLA CORTE, Gabriela; FERNÁNDEZ, Sandra. La metáfora de la región: continente conceptual y construcción historiográfica. Anuario de la Escuela de Historia, Rosario: Universidad Nacional de Rosario, n. 18, p. 149-164, 1997-1998. 
DE CERTEAU, Michel. La escritura de la historia. México: Universidad Iberoamericana, 1993.

DI LISCIA, María Silvia. Dificultades y Desvelos de un Estado interventor. Instituciones, salud y sociedad en el Interior Argentino. La Pampa, 19301946. Anuario IEHS, Tandil: UNCPBA, n²2, p. 93-123, 2007.

Imaginarios y derroteros de la salud en el interior argentino. Los Territorios Nacionales (fines del siglo XIX y principios del $X X$ ). Entrepasados, Buenos Aires, n. 33, p. 49-69, 2008a.

. Reflexiones sobre la 'Nueva Historia Social' de la salud y la enfermedad en Argentina. In: CARBONETTI, Adrián; GONZÁLEZ-LEANDRI, Ricardo (ed.). Historias de salud $y$ enfermedad en América latina siglos XIX y XX. Córdoba: CEA-CONICET, 2008b, p. 15- 47.

FAVARO, Orietta; ARIAS BUCCARELLI, Mario. El lento y contradictorio proceso de inclusión de los habitantes de los territorios nacionales a la ciudadanía política: un clivaje en los años 30. Entrepasados, n. 9, p. 7-26, 1995.

; SCURI, María Carolina. La trastienda de la historia regional. Avances del Cesor, Rosario: ISHIR/CONICET, n. 4, p. 11-18, 2003.

FERNÁNDEZ, Sandra. La Historia sugerente. Los desafíos en la construcción de la historia regional y local. In: MATA DE LÓPEZ, Sara; ARECES, Nidia. (coord.). Historia regional: estudios de casos y reflexiones teóricas. Salta: EdUNSa, 2006, p. 13-22. - Los estudios de historia regional y local de la base territorial a la perspectiva teórico-metodológica. In: FERNÁNDEZ, Sandra R. (comp.). Más allá del territorio: la historia regional y local como problema. Discusiones, balances y proyecciones. Rosario: Prohistoria, 2007, p. 3145.

; DALLA CORTE, Gabriela. (comp.) Lugares para la historia: espacio, historia regional e historia local en los estudios contemporáneos. Rosario: UNR, 2001.

FORCADELL ÁLVAREZ, Carlos. La fragmentación espacial en la historiografía contemporánea: la historia regional/local y el temor a la síntesis. Studia histórica: historia contemporánea, Salamanca: Universidad de Salamanca, n. 13-14, p. 7-27, 1995-1996.

GALLUCCI, Lisandro. Pueblos en contienda. Región y política en el Alto Valle de la Norpatagonia a comienzos del siglo XX. Avances del Cesor, Rosario: ISHIR/CONICET, n. 5, p. 55-72, 2005.

GAUDIO, Ricardo; PILONE, Jorge. El desarrollo de la negociación colectiva durante la etapa de modernización industrial en la Argentina. 1935-1943. Desarrollo Económico. Buenos Aires: IDES, v. 23, n. 90, p. 255-286, 1983. 
HISTORIAPOLITICA.COM. Dossier Interescuelas. Disponible en: http:// historiapolitica.com/interescuelas. Acceso en 3 de nov. 2012.

HORA Roy; TRIMBOLI, Javier. Las virtudes del parricidio en la historiografía. Comentario sobre la mirada de Ema Cibotti a la "generación ausente". Entrepasados, n. 6, p. 89-99, 1994.

KINDGARD, Adriana. M. Procesos sociopolíticos nacionales y conflictividad regional. Una mirada alternativa a las formas de acción colectiva en Jujuy en la transición al peronismo. Entrepasados, n. 22, p. 67-87, 2002.

MONKKONEN, Eric H. The Dangers of Synthesis. American Historical Review, Indiana: American Historical Association, v. 91, n. 5, p. 1146-1157, 1986.

PAGANO, Nora C. La producción historiográfica reciente: continuidades, innovaciones, diagnósticos. In: DEVOTO, Fernando J. (dir.). Historiadores, ensayistas y gran público: la historiografía argentina 1990-2000. Buenos Aires: Biblos, 2010, p. 39-67.

PIAZZESI, Susana. Conservadores en provincia: el iriondismo santafecino 1937-1943, Santa Fe: UNL, 2009.

PONS, Anaclet; SERNA, Justo. Más cerca, más denso. La historia local y sus metáforas. In: FERNÁNDEZ, Sandra R. (comp.). Más allá del territorio: la historia regional y local como problema. Discusiones, balances y proyecciones. Rosario: Prohistoria, 2007, p. 17-30. . Nota sobre la microhistoria. ¿No habrá llegado el momento de parar? Pasado y Memoria: Revista de Historia Contemporánea, Alicante: Universidad de Alicante, n. 3, p. 23, 2004, p. 1-25.

REMEDI, Fernando. La producción en el campo de la historia social argentina en la última década. Historiografías, Zaragoza: Universidad de Zaragoza, n. 1, 2011, p. 53-67, 2011.

REVEL, Jacques. Historia y ciencias y sociales: una confrontación inestable. Estudios Sociales, Santa Fe: Universidad del Litoral, n. 10, p. 11-20, 1996. . Un momento historiográfico: trece ensayos de historia social. Buenos Aires: Manantial, 2005.

ROMERO, Luis Alberto. La historiografía argentina en la democracia: los problemas de la construcción de un campo profesional. Entrepasados, Buenos Aires, n. 10, p. 91-106, 1996.

ROSENTAL, Paul-André. Construire le 'macro' par le 'micro': Fredrik Barth et la microstoria. In: REVEL, J. (dir.). Jeux d' echelles: la micro-analyse à I' experience. Paris: Gallimard-Le Seuil, 1996, p. 141-159.

RUFFINI, Martha. La prensa escrita y la construcción de la figura del ciudadano. Un análisis de las representaciones municipales en el periódico Río Negro. 1912-1917. Revista Pilque, Viedma: Universidad Nacional del Comahue, n. 3, p. 11-36, 2000. 
SABATO, Hilda. La historia en fragmentos: fragmentos para una historia. Punto de Vista, Buenos Aires, n. 70, p. 41-48, 2001.

SERRANO ÁLVAREZ, Pablo. Interpretaciones de la historiografía regional y local mexicana, 1968-1999. Los retos teóricos, metodológicos y líneas de investigación. Revista de História Regional, Ponta Grossa: Universidade Estadual de Ponta Grossa, v. 6, n. 2, p. 113-125, 2001.

SILVA OLARTE, Renán. Del anacronismo en Historia y en Ciencias Sociales. Historia Crítica. Edición especial. Bogotá: Universidad de Los Andes, p. 278-299, 2009.

STEARNS, Peter. Part I: Social History and Spatial Scope. Journal of Social History, George Mason University, v. 39, n. 3, p. 613-614, 2006.

ULLIVARRI, María. Trabajadores, Estado y política durante las gobernaciones radicales en Tucumán. 1935-1943. Anuario del Centro de Estudios Históricos, Córdoba: Centro de Estudios Históricos "Prof. Carlos S. A. Segreti", n. 9, p. 303-321, 2009.

VIALES HURTADO, Ronny J. La región como construcción social, espacial, política, histórica y subjetiva. Hacia un modelo conceptual/relacional de historia regional en América Latina. Geopolítica(s). Madrid: Universidad Complutense, v. 1, n. 1, p. 157-172, 2010. 